\title{
Ontology, methodological individualism, and the foundations of the social sciences*
}

\section{Robert Sugden}

\author{
School of Economics and Centre for Behavioual and Experimental Social Science \\ University of East Anglia \\ Norwich R4 7TJ \\ United Kingdom \\ r.sugden@uea.ac.uk
}

16 September 2015

Abstract: This is a review essay, based on a critical assessment of The Ant Trap by Brian Epstein. Epstein argues that models in the social sciences are inadequate because they are based on a false ontology of methodological individualism, and proposes a new model of social ontology. I examine this model and point to flaws in it. More generally, I argue against Epstein's methodological approach, which treats social ontology as prior to social scientific modelling and as certifying the 'building blocks' that modellers then use. I argue that modellers can legitimately shape the building blocks for their own models.

JEL classification: B40 (economic methodology: general)

Keywords: Ontology, methodological individualism, social fact, collective intention

* This research was supported by the Economic and Social Research Council of the UK (award no. ES/K002201/1.) I thank Stefan Heidl for valuable comments on an earlier version of the paper. 
Brian Epstein has written a book with a mysterious title, The Ant Trap, and an ambitious subtitle, Rebuilding the Foundations of the Social Sciences. It is a work of ontology, the branch of metaphysics that deals with the nature of being. More specifically, it is a contribution to social ontology. At the core of the agenda of social ontology, as understood by Epstein, is the question that he sets out to answer: 'What are social facts, social objects, and social phenomena - these things that the social sciences aim to model and explain?' (7). Many economists will suppose that such an abstruse question has little relevance for their work, however legitimate a concern it may be for philosophers. But Epstein argues that social ontology should be the foundation of the social sciences: 'getting the ontology right' is an essential precondition for sound modelling (278). And that requires the 'sophisticated toolkit of metaphysics' that social ontologists have at their disposal (9).

Epstein is in no doubt that the social sciences in general, and economics in particular, have not got the ontology right, and that, as a consequence of this, their models are inadequate. The Introduction of the book begins with a statement of what Epstein calls 'the Paradox of the Social Sciences'. The supposed paradox is that, over the last twenty years, there has been an explosive growth in the collection of data about people, and so there ought to have been a corresponding improvement in the ability of social scientists to resolve questions about such things as 'the workings of the economy, the sources of poverty, the prescriptions for improving education, and financial regulation'; but in fact the social sciences 'are hardly budging'. The usual clichés about the failure of economists to predict the 2008 crash are rehearsed; the pre-crash optimism of the usual suspects (Ben Bernanke, Oliver Blanchard) is mocked; Paul Krugman's New York Times column is cited in evidence (1-4). After three pages of such commonplaces, Epstein takes the 'failures of social science' to be a matter of established fact. He then tells us that he thinks these failures result from modelling that has been built on unsound ontological foundations. Hence the need to rebuild the foundations of the social sciences.

Where do the ants come in? Epstein's diagnosis of the failures of social science is that the practice of social science rests on a particular analogy between the social and natural sciences. That analogy is that 'that the objects of the social sciences are built out of individual people much as an ant colony is built out of ants'. But this analogy is flawed:

We often think of social facts as depending on people, as being created by people, as the actions of people. We think of them as products of the mental processes, intentions, beliefs, habits, and practices of individual people. But none of this is quite right [...] My aim in this book is [...] to demonstrate that philosophers and 
social scientists have an overly anthropocentric picture of the social world. [The social world] turns out to be not nearly as people-centred as is widely assumed.

(7)

The 'ant trap' is the supposed error of building social science on individualistic foundations. Epstein presents a critique of methodological individualism and proposes a new account of the nature of social facts.

Given Epstein's opening remarks about the failures of social science and his diagnosis of these failures as the result of bad ontology, one might have expected his book to contain some serious discussion of the theories that social scientists actually use. One might have expected to be shown what ontological assumptions are built into these theories, how those assumptions are false, and how theory could be improved by using the ontology that Epstein proposes. In fact, there is almost nothing about any of this. Two major branches of social science theory - game theory and the theory of public choice (or, as it is now sometimes called, political economy) - seem particularly relevant to the kinds of 'social facts' that Epstein discusses, and by implication to be targets of his criticisms. Game theory appears only in a one-sentence footnote, which it has to share with general equilibrium theory (20, note 10). There are occasional brief citations of specific models from public choice theory, but no sustained discussion of the modelling strategy they embody. Nor, incidentally, is there any discussion of how biologists model the behaviour of social animals, and so the claim that the 'ant trap' is a false analogy between social and natural science remains an unsupported assertion. ${ }^{1}$

It is hard to know what to make of these huge gaps in Epstein's argument. The idea that the failures of economics are so obvious as not to need to be demonstrated, and the conviction that these failures can be remedied by abstract philosophical reasoning which takes no notice of existing theory or evidence, express a breathtaking disdain for two hundred and fifty years of work in the discipline. It is perhaps significant that, in Epstein's ontology, facts about human society are fundamentally different from those investigated by the natural sciences - including facts about non-human social animals. This (one might think anthropocentric) distinction allows Epstein to avoid discussing the implications of his method

\footnotetext{
${ }^{1}$ In modelling the behaviour of animals that live in colonies, as ants do, biologists need to be able to track the continuing properties of colonies across generations. One might have thought that such group-level properties might fail to supervene on properties of the individual members of a colony in much the same way that, according to Epstein, supervenience fails for legislatures (see Section 2 below).
} 
of analysis for the natural sciences. But if he is right about the social sciences, ontologists can correct failures in the theories of the natural sciences too, without actually examining them.

As will be clear by now, I found Epstein's discussion of economics unhelpful. But in the remainder of this essay I will overlook the hyperbole of his Introduction. It seems to be intrinsic to Epstein's understanding of the role of social ontology that it discovers the 'building blocks' from which social reality is made, and that good modelling works with these blocks $(9,60)$. Modellers should not be making their own building blocks; they should be using what is already there. If this is right, ontologists can contribute to modelling without knowing anything about models. I propose to read The Ant Trap as an attempt to discover the nature of social reality, and to consider what economists can learn from it. I will begin by considering Epstein's critique of methodological individualism.

\section{Methodological individualism}

Epstein finds the clearest statement of the principle of methodological individualism in the work of the philosopher John W. N. Watkins, a student of Karl Popper. For example:

Now if social events like inflation, political revolution, 'the disappearance of the middle classes', etc., are brought about by people, then they must be explained in terms of people: in terms of the situations people confront and the ambitions, fears and ideas which activate them. In short, large-scale social phenomena must be accounted for by the situations, dispositions and beliefs of individuals. This I call methodological individualism. (Watkins, 1955: 58).

It is surely beyond doubt that the dominant methodology in economics since Adam Smith has looked for explanations of social events in terms of the situations, dispositions and beliefs of individuals. From the late nineteenth century to the present day, most economists have gone further, and looked for explanations of social events in terms of the situations, dispositions and beliefs of rational individuals. Recent developments in behavioural economics have involved some retreat from rationality, but the individualism of classical and neoclassical economics has been retained. The idea that explanations that are based on individual-level assumptions are superior to, or more satisfying than, those that are not is deeply rooted in the practice of economics. (Consider, for example, the positive connotations carried by the idea of 'microfoundations'.)

However, the ontological content of Watkins's statement is contained in the claim that social events must be explained in terms of people. The idea seems to be that, in a 
metaphysical sense, social events just are compositions of individual-level events, and that any genuine explanation of social events must therefore work through individual-level events. Some economists certainly have asserted claims like these as part of what they see as informal motivation for their models, or in describing their methodological positions. It is reasonable to conjecture that many more economists have felt some intuitive attraction to such claims. But that is not to say that the ontology of methodological individualism provides the foundation for modelling in economics.

Epstein (21-35) reviews what philosophers have had to say about methodological individualism in the sixty years since Watkins's statement. He reports that the current 'consensus view' recognises a distinction, first proposed by Steven Lukes (1968), between explanatory individualism and ontological individualism. Explanatory individualism maintains that social facts are best explained in terms of facts about individuals and their interactions, while ontological individualism maintains that social facts are exhaustively determined by facts about individuals and their interactions. The consensus view is that explanatory individualism is a contestable claim about the most useful methodology for social science: it might be true that many social facts are best explained individualistically, but there are no good grounds for treating non-individualistic explanations as unacceptable in principle. In contrast, the consensus view takes ontological individualism to be true - indeed, trivially true, a set of what Lukes (1968: 20) called 'banal propositions'. Epstein summarizes the consensus view as:

We do not need to worry about the ontology of the social world. The social world is nothing but people and their interactions. Of course, we can still fight about methodology. [...] Maybe explanations should be individualistic, maybe not. But the ontology is safe. (23)

Scepticism about explanatory individualism is part of a more general philosophical understanding of the relationship between 'higher-level' and 'lower-level' phenomena. The aggregates that constitute higher-level phenomena often have properties that are not possessed by the lower-level individual items of which they are composed. For example (these are my examples, not Epstein's), a scree slope is a mass of rock debris at the foot of a mountain, constantly being replenished by rock falls. It normally has a constant gradient or angle of repose, which depends on statistical properties of the population of rocks which compose it, but an individual rock does not have a gradient. Highway traffic is an aggregate of moving vehicles. For a given road configuration, the relationship between traffic speed 
and traffic flow has predictable properties that are not properties of the individual vehicles. In cases like these, it is clearly a legitimate scientific aspiration to try to derive higher-level properties from lower-level ones - to 'reduce' the former to the latter. But that is not to say that there is anything wrong with using higher-level properties, such as angles of repose for rock debris or speed-flow relationships for traffic, in explaining other higher-level phenomena. (Indeed, analogies between traffic flows and fluid mechanics have proved useful in transport engineering.) In some cases, even the aspiration to reduce higher-level properties to lower-level ones may be misguided. For example, consider the phenomenon of dorsal fins, as possessed by sharks and dolphins. Considered as large-scale properties of animal bodies, dorsal fins have clearly recognisable properties, connected with their function of assisting the stability of a swimming marine animal; the analogies between the fins of sharks and dolphins are the result of convergent evolution. For some explanatory purposes, it may be more useful to focus on the common function of fins across the species, rather than on their divergent lower-level properties.

Epstein thinks that these (and other) considerations do indeed support a 'maybe, maybe not' position towards explanatory individualism. I agree. But he challenges the second part of the consensus view, that social properties are nothing over and above the properties of individuals.

\section{Supervenience}

In philosophical analyses, the intuitive idea of 'nothing over and above' is usually represented by the concept of supervenience. Epstein offers the following definition, where $A$ is a set of higher-level properties and $B$ is a set of lower-level properties: 'To say $A$ supervenes on $B \ldots$ is to say an object cannot change its $A$-properties without there being some accompanying change in its $B$-properties' (33). For example, a scree slope cannot change its gradient without there being some accompanying change in the properties and positions of individual rocks. Ontological individualism, as construed by Epstein, is the view that 'social facts' supervene on 'individualistic facts'. Epstein argues that this view is false (36).

The difficulty here is that, although supervenience is a well-defined concept, 'social fact' and 'individualistic fact' are imprecise concepts with a wide range of disparate applications. It is one thing to say of a specific social science model that its social properties do or do not supervene on its individualistic properties. Thus, for example, a model of public 
choice might describe a process of pairwise majority voting, and define a concept of 'social preference' in terms of the outcome of this process. In this case, it would clearly be correct to say that, in the model, social preferences supervene on individuals' voting behaviour. But it is another thing to say that, in the real world, the set of all social properties supervenes on the set of all individualistic properties. That assertion seems insufficiently precise to be judged either clearly true or clearly false.

In fairness to Epstein, it must be said that he cites some distinguished scholars who seem to make such claims. For example, Lukes's set of 'banal propositions' includes:

Society consists of people. Groups consist of people. Institutions consist of people plus rules and roles. Rules are followed (or not followed) by people and roles are filled by people. (1968: 120)

In relation to the attribution of agency to groups, Christian List and Philip Pettit (2011: 64) say:

The things a group agent does are clearly determined by the things its members do; they cannot emerge independently. In particular, no group agent can form intentional attitudes without these being determined, in one way or another, by certain contributions of its members, and no group agent can act without one or more of its members acting.

I am not sure that it makes sense to try to show that statements like these are false, rather than merely sententious and imprecise. Nevertheless, it is useful to know what happens if one tries to take ontological individualism at face value. This is what Epstein does in (as he puts it) 'debunking' and 'putting to bed' the claims of ontological individualism $(129,203)$. Although some of the arguments he deploys for this purpose make use of the sophisticated metaphysical toolkit he has told us about, he also uses common-sense arguments which, to my mind, work just as well.

One of his opening examples, intended only to aid intuition, is a question about Starbucks Corporation: Are facts about Starbucks exhaustively determined by facts about its shareholders and its employees? The obvious answer is that they are not:

To be sure, the employees are critical to the operation of Starbucks. But facts about Starbucks seem also to depend on facts about the coffee, the espresso machines, the business license, and the accounting ledgers. (46)

At first sight, this argument might seem to miss the point. Of course, a critic might say, Starbucks would not work without its espresso machines, but it is only the employees who perform actions with those machines. But Epstein can reply that an action is only a special 
kind of operation, and the machines perform operations too. The production of an espresso involves an interaction between the operations of a barista and the operations of an espresso machine. If we are drawing up a list of the lower-level properties on which the higher-level properties of Starbucks supervene, what grounds are there for excluding properties of the machines?

Another example, which Epstein works through in more detail, is an elected legislature (229-233). The legislature is a social entity with an identity that continues over time, even though its membership changes. Since it takes decisions, we can treat it as an agent. $^{2}$ Are facts about its decisions exhaustively determined by facts about its members? One obvious reason for answering 'No' is that the collective decisions of a legislature are determined, not only by how each individual member votes, but also by the rules by which votes are aggregated. Typically, the aggregation rules are not determined by the legislature itself. It does not answer this objection to say, as Lukes does, that 'rules are followed by people' (just as, in the case of Starbucks, ontological individualism is not rescued by saying that espresso machines are operated by baristas). A further problem is that the decisions of a legislature are determined by the votes of its current members, but at any time, the set of members has been determined by the votes of a wider electorate. Thus, viewed over an extended period of time, continuing properties of a legislature's decisions may be determined by continuing properties of the electorate.

Having (as he maintains) shown the falsity of ontological individualism, Epstein concludes that what is needed is more work in social ontology, to find better answers to questions about the nature of social facts. But, one might ask, has the practice of social science actually been harmed by the consensus in favour of ontological individualism? Or is the correct lesson to draw only that social scientists should be more cautious about making grandiose ontological claims?

Epstein thinks the practice of social science has been harmed, but his arguments are unpersuasive - as one might expect, given how little attention he pays to what social scientists actually do. For example, the chapter in which Epstein discusses legislatures ends with a section entitled 'Developing a new perspective on group action'. This new way of

\footnotetext{
${ }^{2}$ In the theoretical framework used by List and Pettit (2011: 19-41), a legislature's continuing identity makes it a 'group', rather than a 'mere collection' of individuals. For it to count as a 'group agent', and so to come within the scope of their version of the principle of ontological individualism, it must also show 'sensitivity to the demands of rationality'.
} 
thinking begins with the recognition that the actions of groups do not necessarily supervene on the actions of group members. That allows us to understand that particular kinds of groups (legislatures being an example) can be set up to achieve particular purposes, and that thousands of years of sociality have endowed human beings with strategies for 'improving the design of groups, helping to ensure that they accomplish their purposes' (234-235). In the case of a legislature, the factors that can be manipulated include its rules for aggregating votes and the rules by which its members are elected. Of course, Epstein is right about this. But if these are new ideas for social ontology, they are not new for social science. They have been at the core of the agenda of public choice since the work of James Buchanan and Gordon Tullock (1962). ${ }^{3}$ Even if economists have sometimes made philosophically questionable claims about the methodological individualism of their work, it does not follow that the substance of that work is unsound.

\section{Collective intentionality}

Alongside his critical discussion of ontological individualism, Epstein considers another philosophical analysis of social facts. This analysis, which Francesco Guala (2007) has called the 'Standard Model of Social Ontology', is particularly associated with John Searle's (1995) analysis of institutional facts. Searle's key formula is ' $X$ counts as $Y$ in $C$ ', where $X$ is a material object, $Y$ is a status that can be assigned to that object, and $C$ is a social context. Such a formula is a constitutive rule. 'Counting as' is understood as the collective recognition, acceptance and acknowledgement of that status within some society. In Searle's central example, $X$ is a U.S. dollar bill, described as a material object, $Y$ is the status 'money', understood as a medium of exchange, and $C$ is 'the United States' (Searle, 1995: 40, 43-51). That dollar bills count as money in the United States is an institutional fact. Searle's interpretation of his formula is:

Collective intentionality assigns a new status to some phenomenon, where that status has an accompanying function that cannot be performed solely in virtue of the intrinsic physical features of the phenomenon in question. (46)

\footnotetext{
${ }^{3}$ Buchanan and Tullock (1962: xvi) describe their approach as 'methodological individualism', which, in the context of public choice, they define by the principle 'Human beings are conceived as the only ultimate choice-makers in determining group as well as private action'. The content of The Calculus of Consent is consistent with this principle, but perhaps not with Epstein's stronger concept of ontological individualism.
} 
Collective intentionality and related concepts, such as plural agency and team reasoning, have been widely discussed by philosophers and have some currency in economics. The common thread in this literature is the idea that two or more individuals can have mental states that relate together in such a way that there is a common attitude (for example, an intention, preference or belief) that each individual can properly attribute to 'us'. Most contributors to this literature have wanted to deny that they were postulating some mysterious form of collective consciousness, and have stressed that such we-attitudes supervene on the mental states of individuals. In this sense, they have endorsed a form of methodological individualism. For example, Searle (1995: 25-26) heads off the criticism that collective intentionality assumes 'some Hegelian world spirit, [...] or something equally implausible' by insisting that his analysis is consistent with the requirements of methodological individualism. Collective intentions are located in the brains of individuals: 'The intentionality that exists in each individual head has the form "we intend".

Epstein presents the Standard Model as a position which, like ontological individualism, is 'almost universally endorsed' by social ontologists. But, he claims, the two positions are in tension with one another: 'Ontological individualism does not logically contradict the Standard Model, but if one is right, it is very likely that the other is wrong' (50-51). However, it turns out that what Epstein means by this is that ontological individualism and the Standard Model represent different relations between individuals and the social world. In other words, we are looking at models of two different kinds of social fact. One kind, exemplified for Epstein by properties of a 'flow of commuters in the Boston metropolitan area, moving in and out of trains, subways and buses', is the focus of ontological individualism. ${ }^{4}$ The other kind, exemplified by properties of a dollar bill, is the focus of the Standard Model (56-57). Epstein seems to be looking for a unified account of the ontology of everything that could possibly be called a 'social fact'; an analysis of a particular type of social fact, however well-defined, is not good enough. ${ }^{5}$ The structure of his

\footnotetext{
${ }^{4}$ This is one of Epstein's examples, but his leading example is 'a mob of drunken hockey fans storming down Howe Street in Vancouver, breaking windows and overturning cars' (56-57). He seems to be thinking of this as a collection of individuals who lack a collective conception of themselves as a mob. But overturning a car requires the concerted action of several people, and is just the kind of case that calls for collective intentionality. Mobs, I would have thought, typically do have collective intentions - for example, to attack a particular out-group, to destroy symbols of an unpopular regime, or (as perhaps for Canadian hockey fans) merely to cause mayhem.

${ }^{5}$ Searle's project is much more limited. He restricts the term 'social fact' to facts that involve collective intentionality, and defines institutional facts - the subject of his analysis - as a subclass of social facts (1995: 26).
} 
own model suggests that he has combined components of ontological individualism and the Standard Model in an attempt to encompass the two kinds of social fact.

\section{Epstein's 'grounding and anchoring' model}

Epstein proposes a model of social ontology whose central concepts are 'grounding' and 'anchoring'. The grounds of a fact $f$ are the elements of the set $\left\{g_{1}, \ldots, g_{m}\right\}$ of 'more fundamental' facts that provides the 'metaphysical reason that $f$ obtains in the world' $(70,82)$. Metaphysical reasons are not causes: roughly speaking, $g_{1}, \ldots, g_{m}$ are what make $f$ the case. In one of Epstein's examples, $f$ is the fact 'The mob ran down Howe Street'; one of the facts that grounds $f$ is 'Bob, Jane, ... and Max ran down Howe Street' (85). A frame principle is a general rule which specifies the grounds for facts of a certain type. Epstein offers the following as an example of a frame principle: 'For all $z$, the fact " $z$ is a bill printed by the Bureau of Engraving and Printing" grounds the fact that $z$ is a dollar' (79).

The concept of anchoring works at a deeper level: 'For a set of facts to anchor a frame principle is for those facts to be the metaphysical reason that the frame principle is the case'. In trying to elucidate this concept, Epstein resorts to metaphors that I find opaque. He starts with the already cloudy concept of a 'natural kind' (roughly, a way of categorising things that is supposed to reflect the structure of the natural world). ${ }^{6}$ By analogy, he talks about 'social kinds'. He says that a kind needs to be held together by some kind of 'glue'. Natural kinds are glued by 'laws of nature' which make things of the same kind behave in regular ways. Anchoring principles are what glue social kinds together. There is an ambiguity here, never resolved by Epstein, corresponding with his distinction between the two kinds of social fact. Sometimes, he seems to be thinking of social kinds as socially accepted categories such as dollars: he says that social kinds 'serve a variety of functions: we employ them for recognizing things, classifying things in various situations, finding and correcting departures from norms...' At other times, staying closer to the analogy with laws of nature, he says that is useful to think of social kinds as 'the categories we might use in the social sciences' (67-69). That suggests that a vortex in the flow of pedestrians in a congested space could be a social kind, even if only one scientist has so far recognised this analogy with fluid mechanics. Epstein excuses the opacity of his discussion of anchoring by saying that

\footnotetext{
${ }^{6}$ Epstein argues that, because we are entitled to assume constancy of properties within natural kinds, natural kinds provide the basis for inductive inference in science (68). I am not convinced that inductive reasoning requires any assumptions about the uniformity of nature (Sugden, 2011).
} 
concepts in metaphysics are difficult to define explicitly, and that what matters is whether the model turns out to be useful (80-82). So let us see how well it works when Epstein applies it to the case of dollars.

Epstein offers a supposedly superior reconstruction of Searle's 'theory of money'. In this reconstruction, the frame principle is the one I have already quoted: 'For all $z$, the fact " $z$ is a bill printed by the Bureau of Engraving and Printing" grounds the fact that $z$ is a dollar'. Epstein calls this principle 'CR' (for 'constitutive rule'). It is anchored by the fact 'We collectively accept CR in the community' (83). Epstein favours this formulation because, he claims, it allows us to distinguish between two ways of representing the requirements of methodological individualism. One is to require that the grounds of a social fact are facts about individuals; the other is to require that the anchors of a social fact are facts about individuals. In Epstein's reconstruction of Searle's formula, facts about dollars are anchored individualistically. In contrast, ontological individualism is a thesis about the grounding of social facts about things such as flows of commuters.

But Epstein seems not to notice that this reconstruction edits out the central idea in Searle's analysis of money. In Searle's ' $X$ counts as $Y$ in $C$ ' formula, $X$ refers to pieces of paper with various special features, including that they are issued by the Bureau of Engraving and Printing. What Americans collectively accept is that these pieces of paper are moneythat, by virtue of their function as a medium of exchange, they have value in America (Searle, 1995: 43-51). In Epstein's reconstruction, Americans collectively accept the much less interesting fact that these pieces of paper are dollars. But, just as the issue of U.S. dollars is governed by institutional rules, so too (presumably) is the issue of Belarusian rubles.

Sufficiently knowledgeable Americans can accept - and, amongst themselves, collectively accept - that pieces of paper that satisfy the Belarusian rules are Belarusian rubles; but that does not make those pieces of paper acceptable in American shops. Notice that this weakness in Epstein's analysis cannot be remedied simply by substituting 'money' for 'dollar'. In that analysis, the fact that some $z$ is a piece of paper issued by the Bureau of Engraving and Printing metaphysically makes it the case that $z$ is a dollar. But the Bureau of Engraving and Printing cannot metaphysically make it the case that its products are acceptable as a medium of exchange in America.

One might think that, in the case of the dollar bill, the set of more fundamental facts that ground the fact ' $z$ is money' include not only facts about the Bureau of Engraving and 
Printing, but also facts about what Americans collectively accept. Epstein rejects this thought as revealing the error of 'conjunctivism' - of treating anchors as grounds.

He presents two arguments against this position (120-124). The first is subtle but, I think, wrong. Suppose I, as a conjunctivist, maintain that what metaphysically makes some piece of paper $z$ money in America is the combination of two facts: (i) that $\mathrm{z}$ is issued by the Bureau of Engraving and Printing, and (ii) that Americans collectively accept the rule that the fact that a piece of paper has been issued by the Bureau metaphysically makes the paper money. Epstein's objection is that this claim asserts that Americans collectively accept a false proposition. They collectively accept that (i) is sufficient to ground the fact that $z$ is money but, according to the claim itself, (i) and (ii) are both required. But this objection fails to recognise the constitutive content of what Searle calls a 'constitutive rule'. ${ }^{7}$ Collective acceptance of a constitutive rule is a joint act that makes the rule a rule. What Searle is imagining is not that each American separately declares his or her private belief that the Bureau of Engraving and Printing metaphysically makes paper into money. Rather, it is as if Americans collectively declare that, by virtue of this declaration, the products of the Bureau are to be regarded by them as money. Such a declaration is self-referential but not (as Epstein seems to think) circular. Its self-referential logic is a familiar feature of exchanges of promises: think of commercial contracts, international treaties, marriages, or indeed constitutions.

Epstein's second argument against conjunctivism is easier to state, but no more convincing as a justification of the model of grounding and anchoring. Characteristically, he formulates conjunctivism as a claim about social facts in general, namely the claim that the grounds of every social fact include the kinds of facts that he calls anchors (115). He then proceeds to argue that 'a given social fact can obtain in possibilities where the anchors for its frame principles do not obtain'. One of his examples, which he sees as 'relatively uncontroversial', is the alleged social fact that Genghis Khan was a war criminal. He says that 'we' (by which I take him to mean present-day citizens of Western democracies) can recognise this as a fact because Genghis Khan 'satisfies the conditions [of being a war criminal] we have anchored' (124). Recalling Epstein's explanation of the concept of a social kind, one might question whether 'war criminal' is a useful category for social scientists trying to explain thirteenth century warfare. Certainly, it is a legitimate scientific

\footnotetext{
${ }^{7}$ It is perhaps revealing that Epstein expresses puzzlement about this term, claiming that Searle's constitutive rules 'are neither constitutive nor are they rules' (77).
} 
enquiry to look for similarities and differences among historical instances of large-scale killing of non-combatants in warfare, and the actions of Genghis Khan might feature in such an enquiry alongside more recent events, such as the mass killing of Jews during the German invasion of the Soviet Union in 1941-42 or the fire-bombing of German and Japanese cities by Allied air forces at the end of the Second World War. But we should not presuppose that the most useful explanatory categories are those that are already recognised by institutions such as the International Criminal Court. The implication, I suggest, is that social scientists are entitled to postulate the existence of social facts that have no anchoring at all (outside the practice of social science itself). I conclude that Epstein's attempt to create a unified ontology of social facts is not succeeding.

\section{Is ontology the foundation of social science?}

Probably Epstein is being too ambitious in trying to find a single model that can encompass the ontology of flows of Boston commuters and the ontology of dollar bills. But, leaving aside the specifics of his model, I want to consider a more fundamental issue: Is an investigation of the ontology of social facts the right way to build the foundations of the social sciences? Or, more fundamentally still: Do the social sciences need foundations at all?

Consider the kind of questions that Epstein is asking. In his analysis of money, he takes the case of a dollar-like piece of paper that he has found in his pocket and asks, in effect: Is this really a U.S. dollar? The answer to this question does indeed depend on whether that paper was issued by the Bureau of Engraving and Printing. Epstein is treating this as a microcosm of the question that social ontology should address: 'What are social facts, social objects, and social phenomena - these things that the social sciences aim to model and explain?' The implication is that an economic model of money that is to be applied to the United Sates needs to be based on a true account of what makes a piece of paper a U.S. dollar. But Epstein's question about the piece of paper is not the kind of question that economists typically ask when they theorise about money. Should it be?

As a thought experiment, suppose that the Mafia has developed a method of producing counterfeit twenty-dollar bills that are not detectably different from the products of the Bureau of Engraving and Printing. It is circumspect in introducing these bills into circulation. As a result, Mafia bosses enjoy lavish lifestyles but there is only a marginal effect on the total money supply. Are these bills really U.S. dollars? Epstein would say 
'No', and that seems to be the right answer. But are they money? I think Epstein has to say that they are not really money. Interestingly, Searle (1995, pp. 32-33) would agree.

Discussing a counterfeit bill, he says that 'people would think it was money even though it was not in fact money'. I think what he means by this is that, according to the constitutive rule that Americans collectively accept, a forged bill does not count as money. That is perhaps right too. But how relevant is all this for an economic model of money as a medium of exchange?

If the Mafia's counterfeit dollar is not detectably different from the real thing, both types of bill will serve interchangeably as a common medium of exchange. A natural way of modelling this scenario - say, as part of an explanation of changes in the general price level in an economy with forgery - would be to use a model in which paper money is a homogeneous commodity, produced both by the Bureau of Engraving and Printing and by the Mafia. In a model of this kind, the concept of 'money' does not correspond with Epstein's or Searle's ontological accounts of what money really is. But in deciding to use this modelling strategy, the modeller does not need to engage in ontological analysis of the true nature of money. The rationale for amalgamating the two types of bill into a common category comes from economic reasoning about the properties of trade and from an understanding of what the model is designed to do. The point of this example is that modelling in the social sciences is not simply a matter of working with building blocks that have been shaped by the abstract reasoning of social ontologists. Modellers often have to shape their own building blocks.

It is an unresolved question in the methodology of economics whether modellers are committed to the claim that their models offer stylized descriptions of certain properties or tendencies of the real world, or merely to the claim that models describe self-contained imaginary worlds that in some respects are similar to the real world. On the first view, espoused for example by Uskali Mäki (1992) and Nancy Cartwright (1998), the concepts used in an empirically successful model might perhaps be interpreted as 'social kinds' in one of Epstein's senses - as categories that are suitable for use in social science. Such kinds, one might say, are glued together by the empirical tendencies that the model exhibits. But that does not mean that those social kinds were recognised as such, prior to the work of the modeller: the modeller may be discovering previously unrecognised social kinds. On the second view, which has been defended by Ronald Giere (1988) in the context of natural science and by me in relation to economics (Sugden, 2000), model-building does not take any stand on the ontology of the real world. 
I do not want to be read as arguing that social ontology has nothing to contribute to economics. To the contrary, it has made a significant contribution to what I (admittedly as an interested party) believe to be a significant development in economics, the theory of team reasoning. The central idea in this theory is that, in certain kinds of interactions within groups of people, each member of the group understands her own action as a component of the joint action of the group; in choosing how to act, each individual asks 'What should we do?' rather than 'What should I do, given what I expect the others to do?' (Sugden, 1993; Bacharach, 2006). This approach has generated insights into how players of pure coordination games identify focal points (Bardsley et al., 2010) and into how people might understand the moral status of market transactions (Bruni and Sugden, 2008). Although the research programme of team reasoning originated in the moral philosophy of act utilitarianism (Hodgson, 1967; Regan, 1980), it has subsequently cross-fertilized with the literatures of collective intentionality and plural agency (Tuomela and Miller, 1988; Gilbert, 1989; Bratman,1993; Searle, 1995). Much of the work in the latter literatures has been motivated by ontological questions about the existence and nature of we-attitudes.

As I understand these literatures, however, they are not concerned with the foundations of social science in the same sense that Epstein is. A characteristic feature of philosophical work on collective intentionality and related concepts is the attention it gives to the details of specific kinds of interaction between small groups of individuals. Paradigm cases include two singers of a duet, two people walking together, two people painting a house together, and two players in a soccer team executing a combination of runs and passes. Perhaps, as Epstein suggests, these accounts of joint action are sometimes 'too idealized and intellectualistic' (255), in something like the way that game theorists' accounts of strategic interaction might be said to be too idealized and rationalistic. Nevertheless, they have provided valuable models of how groups can be considered as agents with intentions and preferences. Good modelling, I submit, requires the modeller to have a clear sense of the concreteness, specificity and credibility of the model world she has constructed, and to find significant similarities between features of that imaginary world and features of the real one (Sugden, 2000). I believe that this is just as true for ontology as it is for economics.

Epstein seems to favour a different methodology. Referring to models of collective intentionality of the kind that I have described, he says that he does not intend 'to devalue the detailed inquiry into more restrictive cases, such as the small-group paradigm', but (proceeding to devalue it) that we must not lose sight of the fact that 'from just one special 
sort of realization, we cannot derive a common set of grounding conditions for group agency' (p. 262). Again we see Epstein's aspiration to create an all-encompassing ontology of social facts. He is right to think that the small-group models about which he is so condescending will not provide ontologically-certified building blocks which social scientists will (or should) then put to use. Rather, these models are contributions to the ongoing research programmes of social science. But that, I think, is all to the good.

If social ontology does not provide the foundations for the social sciences, what does? I commend Otto Neurath's (1937, p. 276) famous metaphor:

We possess no fixed point which may be made the fulcrum for moving the earth; and in like manner we have no absolutely firm ground upon which to establish the sciences. Our actual situation is as if we were on board ship on an open sea and were required to change various parts of the ship during the voyage.

Neurath, as I understand him, is telling us that science has no foundations.

\section{References}

Bacharach, Michael (2006). Beyond Individual Choice: Teams and Frames in Game Theory. Princeton: Princeton University Press.

Bardsley, Nicholas, Judith Mehta, Chris Starmer, and Robert Sugden (2010). Explaining focal points: cognitive hierarchy theory versus team reasoning. Economic Journal 120: 40-79.

Bratman, Michael (1993). Shared intention. Ethics 104: 97-113.

Bruni, Luigino and Robert Sugden (2008). Fraternity: why the market need not be a morally free zone. Economics and Philosophy 24: 35-64.

Buchanan, James M. and Gordon Tullock (1962). The Calculus of Consent. Ann Arbor: University of Michigan Press. Page references to The Collected Works of James M. Buchanan, volume 3 (Indianopolis: Liberty Fund, 1999).

Cartwright, Nancy (1998). Capacities. In The Handbook of Economic Methodology, ed. John Davis, Wade Hands and Uskali Mäki, Cheltenham: Edward Elgar, pp. 45-48.

Gilbert, Margaret (1989). On Social Facts. London: Routledge.

Giere, Ronald (1988). Explaining Science. Chicago: University of Chicago Press. 
Guala, Francesco (2007). The philosophy of social science: metaphysical and empirical. Philosophy Compass 2: 954-980.

Hodgson, David (1967). Consequences of Utilitarianism. Oxford: Clarendon Press.

List, Christian and Philip Pettit (2011). Group Agency: The Possibility, Design, and Status of Corporate Agents. Oxford: Oxford University Press.

Lukes, Steven (1968). Methodological individualism reconsidered. British Journal of Sociology 19: 111-129.

Mäki, Uskali (1992). On the method of isolation in economics. Poznań Studies in the Philosophy of Science and the Humanities 26: 316-351.

Regan, Donald (1980). Utilitarianism and Cooperation. Oxford: Clarendon Press.

Searle, John (1995). The Construction of Social Reality. New York: Free Press.

Sugden, Robert (1993). Thinking as a team: towards an explanation of nonselfish behaviour. Social Philosophy and Policy, 10: 69-89.

Sugden, Robert (2000). Credible Worlds: the Status of Theoretical Models in Economics', Journal of Economic Methodology, 7: 1-31.

Sugden, Robert (2011). Salience, inductive reasoning and the emergence of conventions. Journal of Economic Behavior and Organization, 79: 35-47.

Tuomela, Raimo and Kaarlo Miller (1988). We-intentions. Philosophical Studies 53: 367389.

Watkins, John W. N. (1955). Methodological individualism: a reply. Philosophy of Science 22: $58-62$. 